\title{
Blucher
}

\author{
Blucher Proceedings \\ Cuba e Brasil no Século XXI (CBS21) \\ Inovação e Desenvolvimento Socioeconômico Sustentável
}

\section{Protección de la Propiedad Industrial en Cuba y Brasil.}

\author{
Dra. Marta Moreno ${ }^{1}$, Dra. Silvia Beatriz Beger Uchôa ${ }^{2}$ \\ y Dra. Tatiane Luciano Balliano ${ }^{3}$
}

\begin{abstract}
Generalidades
La Propiedad Intelectual en sus dos acepciones fundamentales, el Derecho de Autor y la Propiedad Industrial, es reconocida y regulada en Cuba y en Brasil. Desde el año 1975 Cuba es miembro de la Organización Mundial de la Propiedad Intelectual (OMPI) como organismo especializado de la Naciones Unidas y parte de los Convenios fundamentales en esta materia, el desde 1904 y el Convenio de Berna de 1886 desde suscrito en el año 1997. Brasil es miembro de la OMPI desde 1975, tiendo suscrito el Convenio de París en 1883 juntamente con otros diez países y el Convenio de Berna de 1886, en 1922.

En materia de Derecho de Autor está vigente en Cuba la ley 14 del año 1977 y en Brasil la ley 9610 del año 1998. Hay gran similitud entre las protecciones das leyes Cubanas y Brasileñas en los aspectos de Derechos de Autor, mas non serón debatidos en este artículo. Una diferencia está en la duración de las facultades patrimoniales, a cual es de toda la vida del autor y cincuenta años post mortem, en correspondencia con lo dispuesto en la Convención de Berna, en Cuba e setenta años en Brasil.
\end{abstract}

\footnotetext{
${ }^{1}$ Profesora Titular. Facultad de Derecho de la Universidad de La Habana (UH). Jefa de la Cátedra de Propiedad Intelectual de la UH. La Habana, Cuba. E-mail: marta.moreno@lex.uh.cu.

${ }^{2}$ Universidade Federal de Alagoas, Maceió, Alagoas, Brasil. E-mail: sbuchoa@gmail.com.

${ }^{3}$ Universidade Federal de Alagoas, Maceió, Alagoas, Brasil. E-mail: .
} 
En Cuba no se dispone en la norma de referencia de forma especial la protección de los programas de ordenador por esta vía, son protegibles a través del derecho de autor, enguanto en Brasil disponemos de la Ley 9609 del año 1998.

A diferencia del Derecho de Autor, la Propiedad Industrial en Cuba si ha sido objeto de modificaciones sustanciales en el orden normativo, a partir de ser miembros fundadores de la Organización Mundial del Comercio como continuadores del Acuerdo General de Aranceles y Comercio (GATT) de 1947 y suscribir el Acuerdo de los Derechos de Propiedad Intelectual relacionados con el Comercio (ADPIC). Desde el propio año 1995 se promulgó el Decreto- Ley 160, a los efectos permitir la presentación de solicitudes de patentes para los productos farmacéuticos y químicos para la agricultura, para cumplimentar lo dispuesto en el Artículo 70.8 del ADPIC. A partir de esta fecha se han promulgado nuevas disposiciones en materia de signos distintivos, indicaciones geográficas, invenciones, modelos de utilidad, modelos industriales, variedades vegetales y topografía de circuitos integrados.

Los derechos de propiedad industrial, en Brasil, referidos a implicar el desarrollo técnico mediante la actividad inventiva y aplicación industrial (Bocchino et al., 2010) se otorgan al titular de las tecnologías industriales y marcas que tratan de promover la creatividad a través de la protección, difusión y uso industrial. Comprende las patentes de invención y de utilidad, marcas, diseños industriales, indicaciones geográficas y la represión de la competencia desleal. A continuación se describen los diferentes tipos de protección clasificadas como propiedad industrial. En Brasil, el Instituto Nacional de Propiedad Industrial (INPI) es la entidad responsable del registro de marcas, diseños industriales, indicaciones geográficas, los programas informáticos y topografías de circuitos integrados, concesiones de patentes y registros de contratos de transferencia de tecnología.

\section{Protección de las Invenciones, Dibujos y Modelos Industriales}

El 1 de abril de 2012 entra en vigor en Cuba el Decreto Ley 290 en materia de Invenciones y Dibujos y Modelos Industriales el cual se aviene a los requerimientos de ADPIC, haciendo gala de las flexibilidades en cuanto a objetos protegibles y además se despoja de instituciones históricas, poco atractivas para solicitantes extranjeros como el Certificado de Autor de Invención el cual reconocía la titularidad de las invenciones al Estado en determinados supuestos contemplados en la legislación anterior. Integra en su texto la protección de las Invenciones, Dibujos y Modelos Industriales y además a los Modelos de Utilidad. Sin embargo aún no se ha puesto en vigor el Reglamento que complementa este importante Decreto Ley. Con el Decreto Ley 290, se implementan una serie de cambios no solo en cuanto a modalidades protegibles sino también en cuanto a forma de protección, vigencia, naturaleza y alcance de los derechos exclusivos, límites a dichos derechos 
e inversión de la carga de la prueba. En el nuevo texto legal incorpora la regulación de los modelos de utilidad, modalidad que no se encontraba regulada en Cuba, protegiendo además a las invenciones y los dibujos y modelos industriales. El elemento más trascendente que incorpora el Decreto Ley en este sentido es la eliminación del certificado de autor regulado en el Decreto Ley 68 de 1983. Este certificado concedía la titularidad al Estado de todas las creaciones obtenidas en el marco de una relación de empleo, así como para determinados objetos de invención reconocidos en el artículo 39 del citado Decreto Ley. No tenía período de vigencia determinado, ni caducaba y no era necesario el pago de tasas para su adquisición y mantenimiento en vigor.

En Brasil, la Ley de Derecho de Propiedad Industrial 9279 (LPI) está en vigor desde el 14 de mayo de 1996, el resultado del trabajo llevado a cabo entre 1989 y 1995, y el que se propone actualmente para la revisión (CAMARA DOS DEPUTADOS, 2013).

De acuerdo con el INPI (INPI, 2014), la patente es "un título de propiedad temporal de una invención o modelo de utilidad, el Estado concede a los inventores, autores u otras personas físicas o jurídicas titulares de los derechos sobre la creación". A cambio, el inventor revela su invención en detalle, con todo el contenido técnico que se contiene dentro de ella. Tiene dos tipos de patentes: la invención y modelos de utilidad. Se considera patentable una invención que cumpla con los requisitos de novedad, actividad inventiva y aplicación industrial, a considerar al modelo de utilidad y ser patentado en cuanto tal, el objeto debe presentar un nuevo formulario o arreglo, para mejorar su rendimiento, que implica actividad inventiva y que tiene aplicación industrial (BRASIL, 1996). Un proceso sólo se puede patentar una invención, modelo de utilidad como siempre.

\section{Requisitos de patentabilidad}

En Cuba quedaron reguladas en los artículos 23 al 25 la novedad, la actividad inventiva y la aplicabilidad industrial. Se establece como excepción a este requisito de la novedad mundial lo definido en el artículo 19 relativo a la prioridad de exposición, la cual se amplía en relación con lo regulado en el Decreto Ley 68. Asimismo el artículo 23.4 del Decreto Ley 290 establece como divulgación inocua que no interfiere la novedad mundial, aquella que se produzca a partir de un acto ilícito contra el solicitante o titular, tal como aparece en muchas legislaciones de América Latina.

En cuanto a la novedad, la legislación sigue al criterio de novedad absoluta y aunque a nuestro juicio el artículo 40 del Decreto Ley 68 definía mejor la novedad, muy bien incorpora el nuevo texto la definición del estado de la técnica. El LPI brasileña presume de manera similar en su articulo 8 quanto a patentabilidad, para ser patentable una invención debe cumplir con los requisitos de novedad, actividad 
inventiva y aplicación industrial. Como novedad, cree que no se considerará divulgación de la técnica de una invención o modelo de utilidad que se produce durante los doce (12) meses anteriores a la fecha doce de presentación o de la prioridad de la solicitud, si se hace:

1. por el inventor;

2. Instituto Nacional de la Propiedad Industrial - INPI por la publicación oficial de la solicitud de patente presentada sin el consentimiento del inventor, en base a la información obtenida de o como resultado de los actos realizados por él; o

3. por terceros sobre la base de información obtenida directamente o indirectamente del inventor o el resultado de sus actos. Este tiempo se conoce como un "período de gracia".

\section{Materia patentable}

Quedó establecido en el artículo 21.2 las invenciones solo para productos y procedimientos quedando de forma expresa y detallada, cada una de las creaciones que no se considerarán invenciones, manteniendo las establecidas por el extinto Decreto Ley 68 e incorporando otras como por ejemplo: los esquemas de trazado de circuitos integrados; los programas de computación, las obras científicas, artísticas y literarias y las creaciones estéticas. En cuanto a las creaciones que no serán patentables, se establecen igualmente con detalle y se mantienen algunas que ya habían sido reguladas por el Decreto Ley 68. Se puede decir que hay un gran avance en la nueva ley, con lo que el sistema de propiedad industrial en Cuba cada vez más cerca de Brasil.

\section{Vigencia}

Se prevé, en Cuba, a diferencia del Decreto Ley 68 que establecía una duración de diez (10) años prorrogables a cinco (5) años, una duración del registro de 20 años, contados desde la presentación de la solicitud, quedando en este aspecto el artículo 42 de nuestra legislación en correspondencia con las exigencias del artículo 33 del ADPIC. En Brasil también es así, en el articulo 40 la LPI dispone un término no menor de diez (10) años para la patente y siete (7) años para una patente de modelo de utilidad, a partir de la fecha de concesión, salvo en el caso de la toma de fuerza se impide procedimiento el examen técnico de la solicitud de pendencia judicial o constatada por razones de fuerza mayor.

\section{La protección de los modelos de utilidad y la protección de los dibujos y modelos industriales}


La incorporación de los modelos de utilidad es algo totalmente novedoso, en tanto, en la legislación cubana no se reconocía la protección de tal modalidad. Quedó definido en el artículo 74 cuáles modelos de utilidad son protegibles en Cuba, quedando recogido de forma diferente a otras legislaciones pues es regulado de forma equiparable a una Invención, siendo exigibles los requisitos de patentabilidad previstos en el texto legal. Se diferencian los modelos de utilidad de las invenciones, según la norma cubana de referencia en tres aspectos fundamentalmente: en el período de vigencia, que es menor, diez (10) años en el caso de los modelos de utilidad y veinte (20) años en las invenciones; en el procedimiento de concesión, y en el nombre que adopta la forma de protección, Certificado de patente en las invenciones y de Registro en los modelos de utilidad. Asimismo, no pueden registrarse como modelos de utilidad los procedimientos y los productos químicos y biotecnológicos. El análisis de los requisitos de patentabilidad tiene el mismo rigor tanto en los modelos de utilidad como en las invenciones. En cambio, en Brasil el plazo de protección de modelo de utilidad es de 15 años de edad y también se llama patente, modelo de utilidad, sin embargo. La semejanza a Cuba, en Brasil no puede invocar la protección de los procesos y procedimientos.

En cuanto a los Dibujos y Modelos Industriales, en Cuba la definición del artículo 91 supera la del artículo 108 del Decreto Ley 68 que aunque protegía las formas bi y tridimensionales no contenía buena técnica en la redacción. Distingue y conceptualiza de forma expresa tanto el dibujo como el modelo industrial. Establece como uno de los requisitos para su protección la novedad. No menciona que sea mundial, aunque de un análisis general de las regulaciones de esta modalidad contenidas en el texto legal, se presume que tiene este carácter, sin embargo, consideramos que debió ser expresa tal mención. Un aspecto superado en esta norma es el requisito de progresividad establecido en el Decreto Ley 68 para los modelos industriales que constituía un error y conllevaba a confusión entre esta modalidad y los modelos de utilidad. Ciertamente es el elemento estético y formal novedoso lo que distingue a los diseños industriales, independientemente que este elemento influya en el funcionamiento del producto. Se mantiene la vigencia de diez (10) años. En Brasil, considera-se original El diseño industrial cuando se traduce en una configuración visual distintiva, en comparación con otros objetos anteriores, tanto en pais como en el mundo. La vigencia del registro es de diez (10) años, renovable por tres (3) períodos sucesivos de cinco (5) años cada uno.

\section{Protección de los Signos Distintivos}

En esta materia en Cuba está vigente desde el año 1999 el Decreto ley 203 De Marcas y Otros Signos Distintivos, el cual derogó al Decreto ley 68 en lo relativo a estas modalidades de la propiedad industrial. Asimismo rige la Resolución 
número 63/2000 Reglamento del Decreto Ley número 203 de mayo del 2000. De acuerdo con el INPI (INPI, 2014b), la marca es "todo signo perceptible visualmente, distintivo que identifica y distingue a los productos y servicios y certifica el cumplimiento de determinadas normas o especificaciones técnicas." En Brasil, la LPI en su artículo 123 define los diferentes tipos de marcas, dividiéndolos en:

1. Marcas productos o servicios: que utilizan para distinguir un producto o servicio de otro idéntico, similar o relacionado, de origen diverso;

2. marca de certificación: aquella usada para dar fe de la conformidad de un producto o servicio con determinadas normas o especificaciones técnicas, en particular con respecto a la calidad, naturaleza, material utilizado y la metodología empleada; y

3. marca colectiva: aquella usada para identificar productos o servicios de los miembros de una entidad.

Leyes de ambas paises definen en cuanto a los tipos de marcas, los signos que pueden constituir marcas permitiendo el registro no sólo a los signos denominativos, figurativos y mixtos sino también a las formas tridimensionales, dejando además la posibilidad de regular en el futuro las marcas sonoras y olfativas.

En Cuba se prevé al igual que lo hizo el Decreto Ley 68 la duración del registro por 10 años, renovables por igual período. En Brasil, la duración del registro es igual, pero puede ser renovado por períodos iguales y sucesivos.

\section{Diagnostico Atual de la Propiedad Industrial de la UH}

- Los proyectos de $\mathrm{I}+\mathrm{D}+\mathrm{i}$ no se respaldan legalmente, en todos los supuestos necesarios, a través de convenios y contratos.

- No se hace uso debido de la información de patentes como fuente tecnológica para la investigación y comercialización de los resultados científicos.

- No se definen adecuadamente las estrategias para proteger los resultados investigativos en Cuba y el extranjero.

- No se realizan las publicaciones científicas en el momento oportuno.

- Presentación de solicitudes de patentes en el extranjero por parte de nuestros investigadores, sin un análisis previo de posibilidades reales y perspectivas

- Falta de planificación y ausencia de financiamiento para el registro de la Propiedad Industrial en Cuba y otros territorios de interés. 
- Insuficiente explotación económica de las patentes.

- En ocasiones caducan las patentes, marcas y demás modalidades de la propiedad industrial concedidas titularidad de la UH, por falta de control y vigilancia de nuestros derechos.

- Falta de una acertada negociación de productos y transferencia tecnológica. o Evaluación incorrecta de los bienes intangibles.

- Aún son bajos los ingresos por ventas de productos que incorporan derechos de propiedad industrial y otras modalidades de transferencia tecnológica.

Estos análisis realizados no son privativos de las universidades sino que aún en nuestro sistema empresarial tanto por razones de incultura en materia de propiedad industrial, como por falta de una debida gestión de nuestros derechos existen estas prácticas incorrectas. Por tal motivo como ya hemos apuntado esta materia es prioridad en todo el proceso de actualización de nuestro modelo económico y social, reflejado en los Lineamientos de la política económica y social del Partido y la Revolución.

\section{Estadísticas Sobre los Derechos de Propiedad Industrial en la UH}

- 35 patentes concedidas. De ellas 18 del Instituto de Ciencia y Tecnología de los Materiales (IMRE) 2 del Centro de Estudios de Proteínas de la Facultad de Biología, 10 del Centro de Estudios de Productos naturales(CEPN) de la Facultad de Química, 2 de la Facultad de Química y 3 del Instituto de Farmacias y Alimentos (IFAL).

- De ellas 7 en régimen de cotitularidad con otros centros.

- Existen 2 patentes en estado de solicitud, 1 del IMRE y 1 de la Facultad de Química.

- 24 marcas de registradas. De ellas 14 del IMRE y 10 del Centro de Biomateriales.

- Existe un grupo de productos protegidos por la vía del secreto industrial.

- Existe 1 modelo de utilidad solicitado por el IMRE.

- En el año 2013 caducaron 6 patentes por falta de pago.

A partir del diagnóstico efectuado se ha propuesto el siguiente procedimiento, el cual ya ha comenzado a implementarse. 
1. Todo proyecto de investigación científica con otras universidades o centros de investigación tanto nacionales o extranjeras debe respaldarse legalmente a través de contratos de coolaboración científica técnica. Dichos contratos serán firmados por la directora de ciencia y técnica de la UH. Ya se han firmado dos convenios marcos de cooperación científico técnica con el Centro de Inmunología Molecular y el centro de Ingeniería genética y Biotecnología.

2. Cada vez que se inicie un proyecto de investigación científica deberán solicitarse, a través de la OTRI los servicios de la Oficina Cubana de la Propiedad Industrial (OCPI) para la consulta de la información de patentes.

3. Deberán firmarse contratos de confidencialidad entre la dirección de las facultades o centros y los profesores e investigadores que participen en investigaciones científicas, a los efectos de preservar el valor comercial de la información no divulgada.

4. Cuando sea procedente se deberán firmar acuerdos de confidencialidad entre la UH y otros centros de investigación o universidades para preservar el carácter de información no divulgada de la información transferida.

5. La estrategia para definir la forma de protección más acertada de los resultados de las investigaciones científicas (patente, secreto, modelo de utilidad, marcas) deberá ser sometida a la consideración del grupo negociador de la OTRI integrado por especialistas de diferentes áreas del conocimiento como economistas, juristas, contadores y científicos de diferentes especialidades.

6. Actualización de los representantes legales tanto a nivel de facultades, centros y OTRI para las solicitudes de derechos de propiedad industrial ante la OCPI y vigilancia del mantenimiento de los mismos. Definición de las funciones de estos representantes.

7. Deberá planificarse anualmente el financiamiento necesario para los registros y mantenimiento de los derechos de Propiedad Industrial en Cuba y en el extranjero.

8. Toda propuesta de negocio para comercializar productos resultados de la ciencia universitaria, transferir derechos de propiedad industrial, o realizar servicios científico técnicos o asistencia técnica deberá ser tramitada por las facultades y centros a través de la OTRI, quien la someterá a la evaluación del grupo negociador y a la dirección jurídica de la UH. El rector determinará quién deberá ser la persona facultada para la firma de estos contratos, según alcance, importancia y complejidad del negocio. 
9. Los investigadores recibirán una remuneración por la explotación económica de los resultados científicos protegidos a través de patente. El \% de dicha remuneración deberá definirse en la normativa interna que deberá promulgar la UH para organizar la utilización acertada de los derechos de propiedad industrial en el marco universitario. Para ello se requiere que previamente se promulgue el Reglamento del Decreto ley 290 sobre invenciones, modelos de utilidad y dibujos y modelos industriales que deberá pronunciarse sobre al procedimiento a seguir para garantizar que se cumpla este derecho ya previsto en el Decreto ley 290 como analizamos anteriormente.

10. Cada centro o facultad deberá tener definida una estrategia diferenciada para garantizar la explotación económica de las patentes, secretos y otros resultados de la ciencia.

11. Se elaborarán e implementarán programas docentes en materia de propiedad industrial a impartirse como asignaturas optativas y electivas en las carreras que lo ameriten, pues solo se imparte como asignatura obligatoria definida en el diseño curricular en la carrera de Derecho.

Para la organización, implementación y control de estas acciones en el orden institucional se creará una oficina de propiedad intelectual subordinada a la OTR e integrada por varios especialistas en esta materia.

En el orden normativo se dictará en breve una resolución rectoral en la UH que reglamente todo este proceso y las obligaciones de las facultades, centros, OTRI e investigadores y profesores en esta materia, a sí como el control efectivo de estas acciones.

En especial el estatuto interno de propiedad industrial incluirá entre otros elementos:

1. Creación de la oficina de propiedad intelectual. Funciones y subordinación.

2. Convenios de colaboración científico técnica con centros de investigación científica y universidades tanto nacionales como extranjeras. Contratos $\mathrm{i}+\mathrm{d}+\mathrm{i}$. Procedimiento para la elaboración y firma de los mismos.

3. Titularidad de las creaciones.

4. Deberes y derechos de los investigadores.

5. Acuerdos de cotitularidad con centros de investigación y universidades.

6. Uso de la información de patentes.

7. Publicaciones científicas. 
8. Acuerdos de confidencialidad.

9. Contratos para comercializar los productos protegidos por la vía de la propiedad industrial y otras formas de transferencia de tecnología. Procedimiento.

10. Planificación de los fondos para trámites de registros de los derechos de propiedad industrial en Cuba y en el extranjero.

11. Destino de los fondos derivados de la explotación económica de los derechos de propiedad industrial. Remuneración a los investigadores.

\section{Procedimentos Aprobados y Resultados en la UFAL}

Cada institución prepara su base a la mencionada Ley de Innovación Tecnologica. El UFAL también tiene su política de innovación, desde 2008, el año de la creación del NIT- UFAL* atraves de la Instrucción Normativa 01/2008 PROPEP/UFAL ( UFAL , 2008 ), que prevé la propiedad y la gestión de los derechos sobre la propiedad intelectual y Innovación en la Universidad Federal de Alagoas y otorga facultades y otras disposiciones. En ella son puntos clave como: la creación de un Comité de Propiedad Intelectual e Innovación, declaración de la misión, los objetivos y las competencias del NIT, disciplina la relación de UFAL con empresas y otras ICTs, y la distribución de los beneficios económicos atraves de la comercialización de los productos procedentes de la propiedad intelectual protegida.

Por otra parte, siguiendo la tendencia nacional, el NIT desde el año 2011 también se convirtió en responsable de la gestión del Programa de Emprendimiento de la UFAL ${ }^{* *}$, incluida la formación de una cultura empresarial más contemporáneo y promover desarrollos innovadores de las actividades de investigación en institución (negocios "science driven"). Los instrumentos para estas acciones de la iniciativa empresarial se basan principalmente en conferencias y cursos de corto/medio plazo, con expertos internos y externos, así como los servicios prestados por sus dos incubadoras de empresas : la incubadora de tecnología basada en Incubal y Nucleo Espaço Gente basada en negocios tradicionales o sociocultural ${ }^{4}$.

En cuanto a los procedimientos de NIT- UFAL, éstas se establecen, a partir de un informe de asistencia, que deberá ir acompañada por la persona que realiza el servicio hasta la finalización del proceso. Por lo tanto, hay un mapeo de entidad de las reivindicaciones, como se puede ver en la Figura 1.

Se observa que la mayor demanda ao NIT- UFAL es relacionada con patentes, lo que concuerda con lo reportado en FORMICT a respecto de las protecciones. La segunda cuestión más buscada trata de las marcas. Se puede concluir que esta

\footnotetext{
4* Para obtener más información sobre el NIT UFAL, consulte http://www.ufal.edu.br/nit. ** Para obtener más información sobre el programa de Emprendimiento UFAL, consulte http : //www.ufal.edu.br/empreendedorismo.
} 


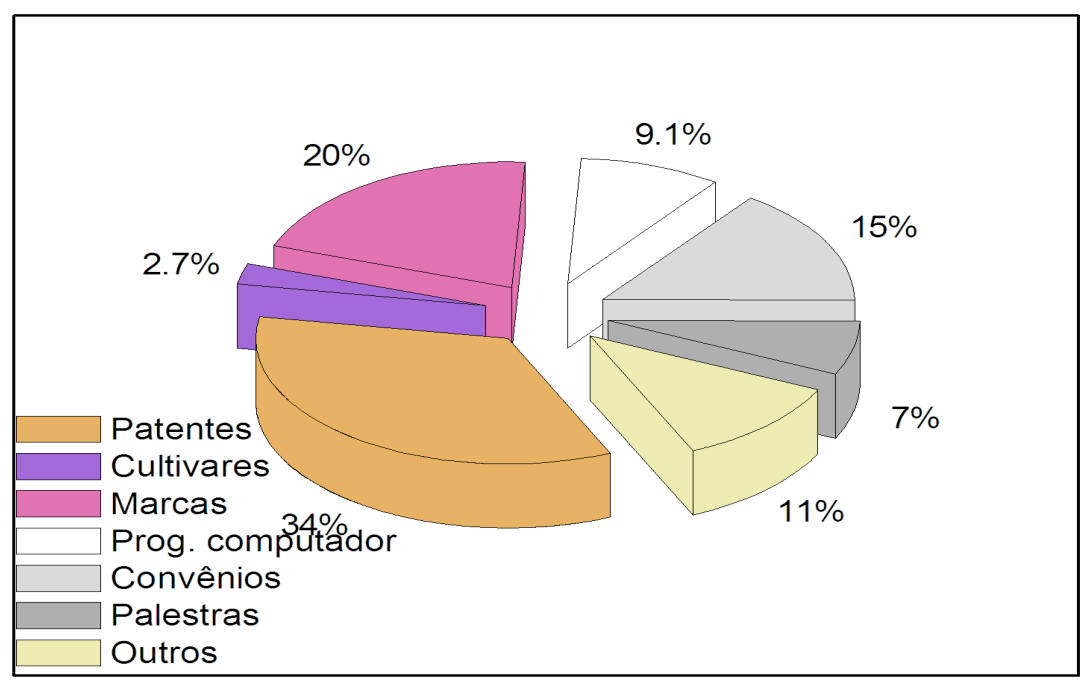

Figura 1: Las demandas ao NIT- UFAL Fuente: elaboración propia

búsqueda es también grande porque el NIT actúa ofecendo apoyar a las empresas incubadas en UFAL bajo la protección de la propiedad intelectual.

A partir de 2008, hubo un aumento en las solicitudes de protección en UFAL debido a la divulgación de los asuntos relativos a la propiedad intelectual y las formas de protección. El UFAL es titular de 23 y cotitular en 4 patentes registradas en el Instituto Nacional de la Propiedad Industrial que todavía tiene la propiedad de 4 depósitos y solicitudes de patentes via PCT e 01 en los Estados Unidos. Puede citar incluso 3 patentes concedidas que aún se encuentran en el nombre del investigador, pero que se presta para la transmisión de la propiedad a UFAL . La figura 2 muestra las solicitudes de protección de la UFAL, realizadas por NIT.

El NIT también actúa sobre la orientación y preparación de los contratos y convenios que celebren entre el UFAL y empresas u otras instituciones de investigación, siempre que el objeto del contrato en materia de propiedad intelectual o la transferencia de tecnología. En este sentido, se realizaron más de 30 llamadas en relación a los convenios, acuerdos y contratos de transferencia de tecnología. También desarrolla actividades relacionadas con los contratos y los acuerdos ya celebrados, como una solicitud de cargos o cambios en los documentos. Se han celebrado 3 contratos de transferencia de tecnología con empresas privadas. También se firmaron 2 acuerdos de confidencialidad para realizar sus actividades y acuerdos de investigación. Los resultados de algunos de estos contratos y acuerdos, se pueden mencionar la recepción de $\mathrm{R} \$ 9.900 .000,00$ en relación con la comercialización de tecnología sin protección. En cuanto a la recepción de una remuneración 


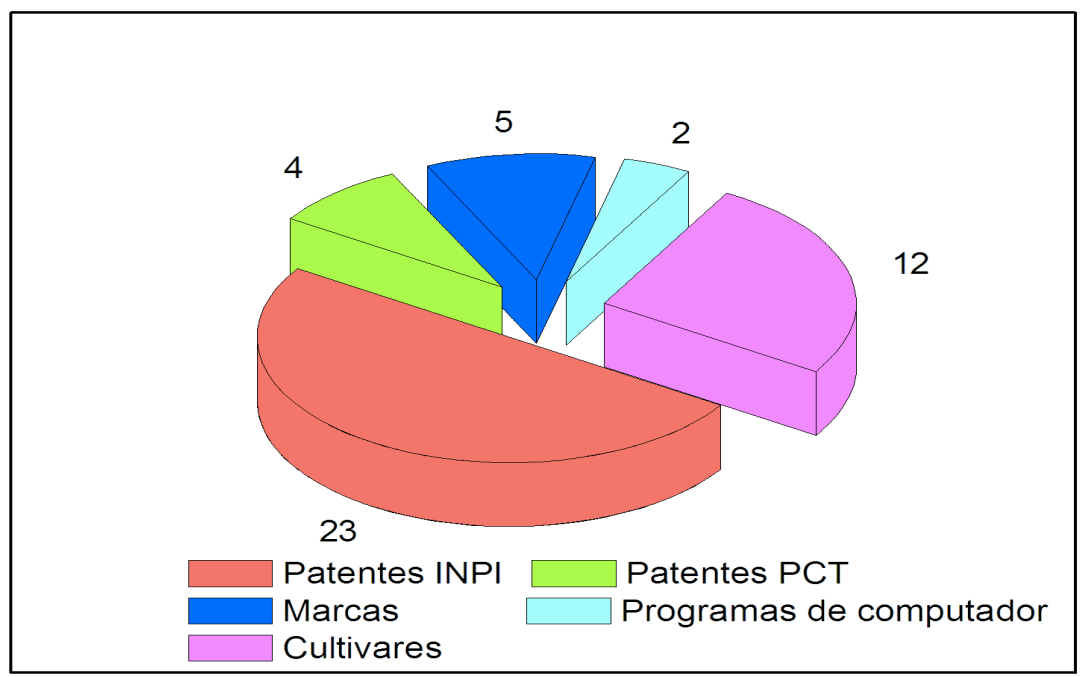

Figura 2: Protecciones celebradas en NIT- UFAL. Fuente: elaboración propia

relativa a un contrato celebrado en el año 2011 , está prevista la recepción de $\mathrm{R} \$$ $26.000,00$ (años 2012 y 2013) en 2014.

\section{Conclusiones}

Conclusiones Elementos importantes fundamentan la prioridad que en los momentos actuales se le concede en Cuba al reconocimiento de la propiedad industrial. Entre ellos se destacan la suscripción de importantes convenios internacionales, en especial el Acuerdo ADPIC de la OMC, la aprobación de políticas encaminadas a poner esta institución al servicio del desarrollo científico, tecnológico, industrial y comercial de nuestro país, incluido el proceso inversionista tanto nacional como extranjero y la aprobación de nuevas normativas atemperadas a nuestro intereses sociales y a la práctica y doctrina internacional.

La legislación brasileña en materia de propiedad industrial buscaba poner al país en el nivel de los países desarrollados. Sin embargo, el desarrollo del sector productivo no siguió este proceso, y que actualmente está discutiendo la revisión de la LPI.

No obstante aún susbsisten en Cuba insuficiencias asociadas a la falta de cultura en esta materia y a una indebida gestión de los activos intangibles como parte del patrimonio empresarial, lo cual conduce en ocasiones a no valorar, proteger, comercializar y defender acertadamente los derechos de propiedad industrial. En 
el orden normativo, se requieren nuevas disposiciones legales o el perfeccionamiento de las existentes en áreas como la información no divulgada, la represión de la competencia desleal, el control del proceso de transferencia tecnológica y la observancia de los derechos intelectuales tanto en el orden administrativo, civil, mercantil y penal.

A partir de las transformaciones actuales del modelo económico y social en Cuba, las universidades cubanas tienen el reto de poner al servicio de la economía sus investigaciones científicas. Asimismo deben garantizar el financiamiento necesario para fomentar el desarrollo de la ciencia aplicada y contribuir a estimular a los creadores e investigadores, protagonistas por excelencia de las creaciones intelectuales. Lo mismo puede decirse en el Brasil, donde tenemos universidades con gran desarrollo en la protección de la propiedad intelectual y la transferencia de tecnología y otros que todavía están empezando.

Para ello se requiere en primer término la realización de investigaciones científicas en función de las principales demandas del sector empresarial, sin obviar por supuesto el desarrollo de investigaciones básicas. Unido a ello se necesita una correcta política y reglamentación de la protección y gestión de las creaciones científicas y su comercialización acertada a partir de los diferentes mecanismos existentes.

Lo anterior no es posible sin una capacitación y especialización del personal vinculado a estas tareas, incluido por supuesto a los investigadores, sin la existencia de interfases que tributen de forma coherente a garantizar los objetivos anteriormente descritos y sin la existencia de normativas internas especializadas que fundamenten legalmente la institucionalidad y el procedimiento a seguir para la debida protección y comercialización de las creaciones científicas universitarias.

Como cierre de este capítulo se puede afirmar que Cuba y Brasil, a pesar de los diferentes sistemas políticos y económicos, ciertas similitudes en los aspectos de la protección de la propiedad intelectual y que UH está muy bien en este aspecto, mientras que UFAL también está jugando su papel.

\section{Referencias}

AMADEI, J.R.P.; TORKOMIAN, A. L. V. As patentes nas universidades: análise dos depósitos das universidades públicas paulistas. Ci. Inf., Brasília, v. 38, n. 2, p. 9-18, maio/ago. 2009

BOCCHINO, L. de O. et al. Propriedade intellectual - conceitos e procedimentos. Brasília: Advocacia-Geral da União, 2010. 316 p. (Série Publicações da Escola da AGU).

BRASIL. Lei n. 9279, de 14 de maio de 1996. Regula direitos e obrigações relativos à propriedade industrial.

Disponível em: www.planalto.gov.br/ccivil 3 3/leis/l9279.htm. Acesso em abr 2014. 
Lei n. 9456, de 25 de abril de 1997. Institui a Lei de Proteção de Cultivares e dá outras providências.

Disponível em: www.planalto.gov.br/ccivil $3 /$ leis/L9456.htm. Acesso em abr 2014.

Lei n. 9609, de 19 de fevereiro de 1998. Dispõe sobre a proteção da propriedade intelectual de programa de computador, sua comercialização no País, e dá outras providências.

Disponível em: www.planalto.gov.br/ccivil $3 /$ leis/l9609.htm. Acesso em abr 2014.

Lei n. 9610, de 19 de fevereiro de 1998. Altera, atualiza e consolida a legislação sobre direitos autorais e dá outras providências.

Disponível em: www.planalto.gov.br/ccivil $3 /$ leis/l9610.htm. Acesso em abr 2014.

Lei n. 10.973, de 02 de dezembro de 2004. Dispõe sobre incentivos à inovação e à pesquisa científica e tecnológica no ambiente produtivo e dá outras providências. Disponível em: www.planalto.gov.br/ccivil ${ }_{0} 3 /$ ato2004-2006/2004/lei/l10.973.htm. Acesso em abr 2014.

Ministério da Ciência, Tecnologia e Inovação. Secretaria de Desenvolvimento Tecnológico e Inovação. Política de propriedade intelectual das instituições científicas e tecnológicas do Brasil: relatório Formict 2012 - Brasília: Ministério da Ciência,Tecnologia e Inovação, 2013.

CAMARA DOS DEPUTADOS. A revisão da Lei de patentes : inovação em prol da competitividade nacional / relator: Newton Lima ; equipe técnica: Pedro Paranaguá (coord.) ... [et al.] ; consultores legislativos: César Costa Alves de Mattos, Fábio Luis Mendes, Mauricio Jorge Arcoverde de Freitas [recurso eletrônico].

- Brasília : Câmara dos Deputados, Edições Câmara, 2013.

INPI. Guia Básico - Indicação Geográfica.

Disponível em: www.inpi.gov.br. Acesso em abr 2014.

(2014a). Guia Básico de Marcas e Manual do Usuário Sistema e-Marcas.

Disponível em: www.inpi.gov.br. Acesso em abr 2014a

(2014b). Guia Básico - Patentes.

Disponível em: www.inpi.gov.br. Acesso em abr 2014.

(2014c). Guia básico - Programa de Computador.

Disponível em: www.inpi.gov.br. Acesso em abr 2014.

LIMA, J. A. DE A. BASES TEORICAS PARA GESTAO DA PROPRIEDADE

INTELECTUAL. Campina Grande: EDUFCG, 2006, 125p.

UFAL . Instrução Normativa 01/2008 - PROPEP/UFAL.

Disponível em www.ufal.edu.br/arquivos/propep/legislacao/nit. Acesso em abr 